\title{
ESTUDIOS
}

\section{Liderazgo y desempeño en la universidad}

\section{Luis Manuel Cerdá Suárez' \\ Casimiro Francisco Ramos ${ }^{2}$}

Resumen: En los últimos años se asiste a un creciente interés por evaluar la calidad de la Enseñanza Superior, como elemento central del nuevo marco educativo. Sin embargo, a pesar de los avances producidos recientemente en la docencia universitaria, orientados hacia un aprendizaje autónomo del estudiante, aún es frecuente encontrar estilos de enseñanza en los que el profesor es el responsable principal del buen desempeño de la clase.

Este trabajo ofrece una herramienta para evaluar la aplicación por el profesor de distintos estilos de liderazgo en el proceso de aprendizaje de los estudiantes. Su principal contribución consiste en avanzar hacia la puesta en práctica de un modelo de gestión en el aula, que supere las limitaciones de las técnicas tradicionales basadas en la clase magistral, y que se ha aplicado en el ámbito de un Convenio Internacional de cooperación científica y técnica, en Universidades de dos países.

Palabras clave: Aprendizaje, competencias, liderazgo, desempeño, docencia, Universidad.

Fecha de recepción: 24 de mayo de 2011 .

Fecha de aceptación definitiva: 27 de junio de $201 \mathrm{I}$.

\footnotetext{
' Facultad de Ciencias Económicas y Empresariales - ETEA, Universidad de Córdoba.

${ }^{2} \mathrm{IADE}$ - Lisboa.
} 


\section{Leadership and University performance}

\begin{abstract}
In recentyears we have witnessed a growing interest in evaluating the quality of Higher Education as a central element in the new educational framework. However, despite the progress recently made in university teaching, oriented to autonomous learning, it is still common to find teaching styles where the teacher is who is primarily responsible for good class performance. This work provides a tool to assess different styles of leadership used by teachers for the students' learning process. Its main contribution is to further the implementation of a classroom management model, that overcomes the limitations of traditional techniques based on the lecture, and that has been applied in the context of an international agreement on scientific and technical cooperation in universities in two countries.
\end{abstract}

Key words: Learning, skills, leadership, performance, teaching, university.

\section{Leadership et rendement à I’université}

Résumé: Nous assistons dernièrement à un intérêt croissant pour évaluer la qualité de l'Enseignement Supérieur, en tant qu'élément central du nouveau cadre éducatif. Cependant et malgré les progrès effectués récemment dans l'enseignement universitaire, orientés vers un apprentissage autonome de la part de l'étudiant, il est pourtant commun de retrouver des styles d'enseignement dans lesquels le professeur est le responsable principal du bon rendement des classes.

Ce document nous offre un outil pour évaluer I'application par le professeur des différents styles de leadership dans le processus d'apprentissage des étudiants. Sa contribution principale étant $d$ 'avancer vers la mise en pratique d'un modèle de gestion en cours, et qui s'est appliqué dans le cadre d'un Accord International de coopération scientifique et technique, dans les Universités de deux pays différents.

Mots clés: Apprentissage, compétences, leadership, rendement, enseignement, Université.

\section{Introducción}

En la última década, se están produciendo importantes cambios en el ámbito de la enseñanza universitaria. El nuevo marco que establece el Espacio Europeo de Educación Superior constituye el referente clave para el desarrollo de un aprendizaje basado en competencias, frente a la alternativa tradicional de transmisión de conocimientos. Así, tras recibir sus títulos correspondientes, los egresados han de incorporarse al mercado de trabajo, y la práctica profesional requiere de ellos que diseñen y comercialicen productos, defiendan los intereses de sus clientes, 
se introduzcan en la Administración Pública o, incluso, que inicien una carrera académica. Las Escuelas y Facultades han de formar a futuros líderes sociales y de opinión, consejeros, asesores, etc.; en definitiva, personas con capacidad para resolver problemas (Atencio y Arrieta, 2005; UNESCO, 1998). Además, los futuros profesionales han de estar capacitados para trabajar en un mundo complejo, en el que las demandas de la sociedad son profundamente cambiantes. Por ello, los alumnos universitarios han de adquirir una variedad de capacidades técnicas, interpersonales e intelectuales que debe atender el currículo académico de las instituciones universitarias (Robbins, 2004; cit. en Bennetts, 2007).

Aunque muchas cuestiones deban ser tenidas en cuenta en el proceso de aprendizaje de los estudiantes, este estudio se limita a desarrollar un método para describir la conducta del profesor en el aula, lo que permitiría relacionar sus distintos patrones de comportamiento con la efectividad en el aprendizaje en los grupos que lidera, en una amplia variedad de situaciones (Fuentes, 2007).

Dentro de esta línea de trabajo, esta investigación partió de una iniciativa de innovación docente, sustentada en los siguientes pilares:

1. La adaptación del proceso de aprendizaje de los alumnos a las nuevas tendencias en metodología docente.

2. La complementación del sistema de enseñanza de clase magistral con nuevas técnicas, basadas en el desarrollo de competencias y en las experiencias de los estudiantes; $y$

3. El trabajo conjunto entre profesor y alumnos, a través de una interacción permanente en el proceso de aprendizaje.

El proyecto pedagógico que se describe en estas páginas ha dado lugar en los últimos años a diversas publicaciones relacionadas (Cerdá y Ramírez, 2010; Cerdá y Ramos, 2011). Forma parte de una investigación comparada de carácter internacional, implantada a nivel de postgrado en dos universidades que, desde hace tiempo, aplican metodologías basadas en competencias: la Universidad Autónoma de Baja California (México) y el Instituto de Artes Visuais, Design e Marketing (Portugal); y se realizó al amparo del Programa de Incorporación de Doctores Españoles a Universidades Mexicanas, establecido entre la Agencia Española de Cooperación Internacional y la Asociación Nacional de Universidades e Instituciones de Educación Superior, de México.

Este artículo se estructura de la siguiente forma: primero se revisa la literatura sobre el liderazgo docente y su desempeño; luego se explica que, debido a la oportunidad 
detectada en la literatura de investigar sobre estos conceptos, resulta apropiado generar procedimientos sencillos y sistemáticos para describir el comportamiento del profesor en el aula. Después se presenta la metodología del estudio empírico, que incluye información detallada sobre los datos, los análisis estadísticos y la interpretación de los resultados. Y se concluye con las contribuciones metodológicas, las implicaciones prácticas, las limitaciones y las futuras líneas de investigación.

\section{Revisión de la literatura}

\section{I. Estilo de liderazgo docente}

En la literatura académica, el liderazgo ha sido objeto de numerosos estudios, desde enfoques muy variados: las teorías de los rasgos y caracteres, conductuales, de contingencias, situacionales, transaccionales y transformacionales. Como resultado de esta diversidad de aproximaciones teóricas, en los últimos años han surgido distintos planteamientos, como los relacionales o los referidos al liderazgo facilitador, persuasivo, carismático, sostenible o visionario (Bennetts, 2007; De Vincenzi, 2009; Pedraja-Rejas et al., 2009); lo que en la práctica hace controvertidos los tópicos de interés en la investigación sobre el liderazgo (Elton, 2001; Lupano y Castro, 2008).

Las investigaciones en este ámbito se han centrado en analizar si el estilo de liderazgo predominante en los docentes se ajusta a uno transaccional -el profesor intercambia calificaciones y recompensas por el esfuerzo de los alumnos- o transformacional -los docentes motivan, estimulan la capacidad analítica de los estudiantes y les ayudan a lograr sus objetivos (Bass y Avolio, 1997 y 2000; Gil-García et al., 2008; González y González, 2008). Aunque los estudios sugieren que hay que huir de enfoques centrados en la existencia de un profesor ideal (hay buenos profesores con rasgos de personalidad y estilos muy diversos, e igualmente eficaces), gran parte de la literatura destaca la importancia de un liderazgo transformacional (Ahumada et al., 2008; Cuadrado et al., 2003; Muenjohn y Armstrong, 2008). Su razón de ser descansa en las siguientes premisas: 1) La pedagogía actual entiende el aprendizaje como un proceso que entraña un cambio constante en la forma de conocer, sentiry actuar del estudiante, superando la concepción tradicional de este como sujeto pasivo de la enseñanza (Biggs y Tang, 2007); y 2) En el aprendizaje de los alumnos, resulta esencial la figura del docente: su influencia depende tanto de su estilo, como del contexto situacional en el que opera. Por este motivo, lo que 
constituye un liderazgo efectivo en determinada situación puede resultar inefectivo en otras (Cerdá y Ramírez, 2010; Elıon, 2001).

En líneas generales, la revisión de la literatura refleja que un mismo grupo puede comportarse de forma diferente, según el liderazgo docente que se ejerce sobre él. En este sentido, se defienden estilos participativos por razones de motivación, satisfacción y eficacia grupales (Caligiore y Díaz, 2006; Cuadrado et al., 2003). El cuadro 1 que se ofrece a continuación presenta la revisión de la literatura sobre este tópico, cuyos rasgos principales se presentan en diversas áreas de actuación docente: destacando la importancia de fomentar la creatividad/experimentación de los alumnos en el aula (con la aparición de nuevos enfoques para viejos problemas), alcanzar la motivación (esto es, la capacidad de entusiasmar, transmitir confianza y respeto a los alumnos) y desarrollar la empatía (por ejemplo, al tratar individualmente al estudiante); en línea con tres ámbitos de trabajo del profesor efectivo: disciplinar (el docente ha de desempeñarse como innovador y facilitador del conocimiento), pedagógico (capacidad del profesor de transmitir motivación y entusiasmo) y personal (como tutor del proceso de aprendizaje de los estudiantes) (Havnes y Mc Dowell, eds., 2007; Hounsell et al., 2007; Murillo, 2006).

\section{CUADRO I. Rasgos principales del estilo de liderazgo docente, y autores más relevantes}

\begin{tabular}{|l|l|}
\hline \multicolumn{1}{|c|}{$\begin{array}{c}\text { INDICADORES DE ESTILO DE LIDERAZGO } \\
\text { DOCENTE }\end{array}$} & \multicolumn{1}{|c|}{ AUTORES } \\
\hline $\begin{array}{l}\text { El profesor proporciona formas nuevas de hacer las } \\
\text { cosas }\end{array}$ & $\begin{array}{l}\text { Bennetts (2007), Biggs y Tang (2007), } \\
\text { Feixas (2004) }\end{array}$ \\
\hline $\begin{array}{l}\text { El profesor ayuda a analizar los problemas desde dife- } \\
\text { rentes puntos de vista }\end{array}$ & Francis (2006), Moss y Ritossa (2007) \\
\hline $\begin{array}{l}\text { El profesor genera nuevas ideas para la solución de los } \\
\text { problemas }\end{array}$ & $\begin{array}{l}\text { Ahumada et al. (2005), De Vincenzi } \\
\text { (2009) }\end{array}$ \\
\hline $\begin{array}{l}\text { El profesor estimula la tolerancia a las diferencias de } \\
\text { opinión }\end{array}$ & $\begin{array}{l}\text { Antonakis et al. (2003), Atencio y } \\
\text { Arrieta (2005), Caligiore y Díaz (2006) }\end{array}$ \\
\hline $\begin{array}{l}\text { El profesor tiende a hablar con entusiasmo sobre las } \\
\text { metas a alcanzar }\end{array}$ & $\begin{array}{l}\text { Bass y Avolio (1997), De Vincenzi } \\
\text { (2009), Ramirez y Sgambatti (2008) }\end{array}$ \\
\hline El profesor me ayuda siempre a que me esfuerce & $\begin{array}{l}\text { Escribano (1995), González (2008), } \\
\text { Ramsdem (1991) }\end{array}$ \\
\hline $\begin{array}{l}\text { El profesor concede elogios cuando se hace un buen } \\
\text { trabajo }\end{array}$ & Biggs y Tang (2007), González (2008) \\
\hline
\end{tabular}




\begin{tabular}{|l|l|}
\hline $\begin{array}{l}\text { El profesor expresa confianza en que se alcanzarán } \\
\text { las metas }\end{array}$ & $\begin{array}{l}\text { Padilla (2002), Pérez et al. (2009), } \\
\text { Zabalza (2000) }\end{array}$ \\
\hline $\begin{array}{l}\text { El profesor tiene en cuenta las consecuencias morales y } \\
\text { éticas de las decisiones adoptadas }\end{array}$ & $\begin{array}{l}\text { Hounsell et al. (2008), Lupano y Castro } \\
\text { (2008), Mandell y Pherwani (2003) }\end{array}$ \\
\hline $\begin{array}{l}\text { El profesor presta atención a sentimientos y necesidades } \\
\text { de los alumnos }\end{array}$ & $\begin{array}{l}\text { Li y Kaye (1998), Lowe et al. (1996), } \\
\text { Zabalza (2000) }\end{array}$ \\
\hline El profesor se relaciona conmigo personalmente & $\begin{array}{l}\text { Fuentes (2007), Gil-García et al. } \\
\text { (2008), Joseph y Joseph (2007) }\end{array}$ \\
\hline $\begin{array}{l}\text { El profesor me trata como individuo y no como miembro } \\
\text { de un grupo }\end{array}$ & $\begin{array}{l}\text { Navaridas (2004), Pascual et al. } \\
\text { (2006), Pedraja-Rejas et al. (2009) }\end{array}$ \\
\hline El profesor dedica tiempo a orientar y enseñar & UNESCO (2002), Zabalza (2000) \\
\hline
\end{tabular}

Elaboración propia.

\subsection{Desempeño del profesor en el aula}

Diversas investigaciones han concluido que el estilo de liderazgo tiene un impacto significativo sobre el desempeño en distintos ámbitos. En particular, el liderazgo en el terreno pedagógico se refiere a la capacidad del docente para crear un clima en el aula que favorezca el aprendizaje, a través de la satisfacción, los esfuerzos y la eficacia de los estudiantes en su desarrollo académico (Antonakis et al., 2003; Pérez et al., 2009). Para lograr esto, resulta oportuno experimentar en la clase dicha atmósfera, fomentando la participación de los alumnos y su responsabilidad en el proceso de aprendizaje.

El clima en el aula puede entenderse como "el ambiente interno de la clase que no es físicamente palpable, pero que se siente, de carácter multidimensional" (Caligiori y Díaz, 2006; Pérez et al., 2009). Aunque en el ámbito organizativo este concepto se ha analizado desde hace ya mucho tiempo, en el terreno pedagógico las investigaciones son más recientes. Así, los modelos conceptuales y los diferentes enfoques que analizan la complejidad de la vida en el aula han evolucionado desde la racionalidad técnica-que explica las prácticas docentes mediante patrones de conducta esperables, ante situaciones regulares-, hasta una visión constructivista -que defiende la singularidad y complejidad de la clase (De Vincenzi, 2009; Escribano, 1995; UNESCO, 1998).

La importancia del clima generado en el aula reside en que su ambiente desinhibido refuerza una participación activa de los alumnos en el proceso de aprendizaje (Escribano, 1995; Feixas, 2004). Así, diversas investigaciones sostienen que las 
habilidades interpersonales y el comportamiento del profesor influyen sobre el éxito en el aprendizaje de los estudiantes (Campos et al., 2001; Navaridas, 2004; Padilla, 2002); aunque otras evidencias son menos concluyentes al respecto (Berggren et al., 2005; Boyatzis, 1999; Dochy et al., 1999; Joseph y Joseph, 1997; Pedraja-Rejas et al., 2009).

Admitiendo que un liderazgo docente participativo refuerza el aprendizaje de los alumnos, el desempeño del profesor en el aula proporciona información sobre qué y cómo se está aprendiendo (AC Nielsen, 2000; Biggs y Tang, 2007). En el cuadro 2 se muestran distintos indicadores sobre criterios de evaluación del desempeño docente: los relativos a la satisfacción obtenida por los estudiantes (entendida como un ambiente sano para el buen desempeño del grupo); conseguir de los alumnos un esfuerzo extra (es decir, una mayor participación en su proyecto formativo) y lograr la efectividad del proceso de aprendizaje (las acciones del profesor permiten lograr, de forma armónica, los objetivos programados en el curso).

\section{CUADRO 2. Principales criterios de evaluación del desempeño docente, $y$ autores más relevantes}

\begin{tabular}{|c|c|}
\hline $\begin{array}{l}\text { INDICADORES DE DESEMPEÑO DEL } \\
\text { DOCENTE }\end{array}$ & AUTORES \\
\hline $\begin{array}{l}\text { El profesor utiliza métodos didácticos que me } \\
\text { resultan satisfactorios }\end{array}$ & $\begin{array}{c}\text { Antonakis et al. (2003), Biggs y Tang (2007), } \\
\text { Ramsdem (1991) }\end{array}$ \\
\hline $\begin{array}{l}\text { El profesor mejora el clima en el aula con buen } \\
\text { humor }\end{array}$ & Castillo y Cabrerizo (2003), González (2008) \\
\hline $\begin{array}{l}\text { El profesor se muestra satisfecho con la partici- } \\
\text { pación y logros del grupo }\end{array}$ & AC Nielsen (2000), Biggs y Tang (2007) \\
\hline $\begin{array}{l}\text { El profesor expresa satisfacción cuando cumplo } \\
\text { con lo esperado }\end{array}$ & Elton (2001), Havnes y McDowell (eds., 2007) \\
\hline $\begin{array}{l}\text { En general, estoy satisfecho con el estilo docente } \\
\text { del profesor }\end{array}$ & $\begin{array}{c}\text { Havnes y McDowell (eds., 2007), Pedraja- } \\
\text { Rejas et al. (2009) }\end{array}$ \\
\hline $\begin{array}{l}\text { El profesor me motiva a trabajar más duro en } \\
\text { lo que hago }\end{array}$ & $\begin{array}{c}\text { Fuentes (2007), González y González (2008), } \\
\text { Lupano y Castro (2008) }\end{array}$ \\
\hline $\begin{array}{l}\text { El profesor me motiva a hacer más de lo que } \\
\text { esperaba }\end{array}$ & $\begin{array}{l}\text { Ávila (2005), Feixas (2004), Joseph y Joseph } \\
\text { (2007) }\end{array}$ \\
\hline $\begin{array}{l}\text { El profesor me hace sentir orgullo por el trabajo } \\
\text { bien hecho }\end{array}$ & $\begin{array}{c}\text { Murillo (2006), Navaridas (2004), UNESCO } \\
\text { (1998) }\end{array}$ \\
\hline
\end{tabular}




\begin{tabular}{|c|c|}
\hline $\begin{array}{l}\text { El profesor nos recompensa por los logros al } \\
\text { alcanzar las metas }\end{array}$ & Bennetts (2007), Francis (2006) \\
\hline $\begin{array}{l}\text { El profesor aumenta en general mi motivación } \\
\text { por tener éxito }\end{array}$ & $\begin{array}{c}\text { Mueniohn y Armstrong (2008), Parker et al. } \\
\text { (2004) }\end{array}$ \\
\hline $\begin{array}{l}\text { El profesor es efectivo en buscar formas de } \\
\text { motivar al grupo }\end{array}$ & $\begin{array}{l}\text { Berggren et al. (2005), Escribano (1995), Pérez } \\
\text { et al. (2009) }\end{array}$ \\
\hline $\begin{array}{l}\text { El profesor es efectivo en satisfacer las necesidades } \\
\text { del grupo }\end{array}$ & Ramirez y Sgambatti (2008) \\
\hline $\begin{array}{l}\text { El profesor es efectivo al asignar tareas para } \\
\text { lograr los objetivos }\end{array}$ & Hounsell et al. (2007) \\
\hline $\begin{array}{l}\text { El profesor está dispuesto a ayudar siempre que } \\
\text { se le necesite }\end{array}$ & Biggs y Tang (2007), Dochy et al. (1999) \\
\hline $\begin{array}{l}\text { El general, estoy satisfecho con el cumplimiento } \\
\text { de los objetivos del curso }\end{array}$ & $\begin{array}{l}\text { Boyatzis (1999), Campos et al. (2001), Lowe et } \\
\text { al. (1996) }\end{array}$ \\
\hline
\end{tabular}

Elaboración propia.

\subsection{Un enfoque de gestión del conocimiento}

Numerosos estudios han identificado la gestión del conocimiento como un aspecto clave en el desarrollo exitoso de las empresas (Prahalad y Hamel, 1994; Levitt y March, 1998), debido a que una organización que dirige bien el conocimiento tiene el potencial de crear un valor diferencial, vinculado con su estrategia de actuación en el mercado (Davenport y Prusak, 1998; Nonaka, 1995; Polanyi, 1996; Prieto, 2006). Su aparición en el campo de la Dirección de Empresas supuso un hito importante, porque integró muchos de los conceptos manejados anteriormente en este ámbito. Las conclusiones derivadas a partir de este enfoque se han utilizado para fines múltiples, y han contribuido también al desarrollo de modelos de gestión en el aula.

La aplicación de la gestión del conocimiento a nuestro tema de investigación ha permitido conocer mejor los patrones de comportamiento del profesor. Diversas investigaciones sostienen que este tipo de aprendizaje es una forma eficiente de alcanzar los resultados docentes, al implicarse los estudiantes en una experiencia y reflexionar sobre ella (Frontczak y Kelley, 2000; Gentry, 1990; Kolb, 1984). El liderazgo transformacional se explica mejor por este enfoque, ya que propone fomentar una cultura en el aula que facilite y valore un aprendizaje en y a través de las personas (Frontczak y Kelley, 2000; Hamer, 2000). Los seminarios, y a través de ellos los debates, constituyen ejemplos de técnicas activas de aprendizaje, por 
las ventajas de una mayor implicación del alumno en este proceso (Bobbit et al., 2000; Kennedy, Lawton y Walker, 2001). La literatura pedagógica señala que los profesores que han incorporado este tipo de metodologías han constatado un elevado grado de identificación y entusiasmo entre los estudiantes (Burns, 1990; Hamer, 2000).

\section{La aplicación de un modelo de gestión en el aula}

Aunque diversas investigaciones han concluido que el liderazgo transformacional es el mejor enfoque para evaluar el desempeño docente, no abundan las escalas únicas sobre esta estrategia didáctica: las aportadas por muchos estudios tienen poca utilidad práctica para actuar en el aula, ya que no permiten representar el fenómeno estudiado en sus dimensiones operativas.

Para la medición del estilo de liderazgo se emplean habitualmente escalas multidimensionales, como la propuesta por Bass y Avolio (1997), aunque varias investigaciones señalan diversas limitaciones de este tipo de instrumentos. Por ejemplo, que su estructura factorial no es universal: algunos factores se subdividen entre sí, mientras que otros desaparecen (Cuadrado et al., 2003; Pascual et al., 2006); o que los datos utilizados proceden de muestras relativamente pequeñas. Además, aunque hay evidencias de que el enfoque transformacional logra un mejor desempeño en el aula, no abundan las escalas únicas de estilos transformacionales: las aportadas por algunos estudios se refieren, al mismo tiempo, a distintos estilos de liderazgo.

Diversos autores recomiendan avanzar en el conocimiento del tipo de liderazgo que se requiere en el contexto actual, y perfeccionar las escalas de liderazgo, mediante procedimientos sencillos para facilitar la toma de decisiones en el aula (Lowe et al., 1996; Mbawmbaw et al., 2006; Moss y Ritossa, 2007). En particular, resulta oportuno integrar el estilo de liderazgo del profesor con información sobre su desempeño; tener en cuenta el clima generado por aquel en el aula, y desarrollar protocolos de actuación aplicables en este ámbito (Berggren et al., 2005; Castillo y Cabrerizo, 2003).

Para cubrir la laguna existente en la literatura, hemos diseñado un procedimiento basado en un estilo participativo de gestión en el aula, mediante una escala original de medición del liderazgo docente, junto con la evaluación de su desempeño; con el propósito de que se utilice como una herramienta para la mejora académica. 


\section{Metodología}

\section{I. Ámbito de estudio}

Para analizar la importancia del estilo de liderazgo del profesor y evaluar su desempeño, se aplicó una investigación en México y en Portugal. Tres aspectos de la metodología deben señalarse en este estudio: primero, en relación con la población investigada; en segundo término, en cuanto a la aplicación de la estrategia de aprendizaje en el aula. Y, en tercer lugar, respecto de las fuentes de información y el procedimiento detallado de la investigación, como se expone en el apartado siguiente.

En primer lugar, con el fin de explorar estos constructos se experimentó en México sobre dos grupos de alumnos, en el postgrado de Administración y de Tecnologías de la Información. En ambos casos, todo fue igual menos la exposición a dos estilos de profesores, contrapuestos entre sí en cada grupo y definidos sobre tres parámetros (para caracterizar una metodología participativa de otra que no lo es): a) filosofía docente (los objetivos se fijan -o no- en consenso entre profesor y alumnos); b) autonomía (libertad -o no- del grupo al decidir el contenido concreto de su asignatura); y c) relación con el alumno (disposición personal -o no- del profesor con el estudiante).

En segundo lugar, se puso en práctica una estrategia de aprendizaje basada en el desarrollo de seminarios, mediante trabajos y presentaciones en grupo. En el grupo sometido a metodologías participativas se ensayaron distintas técnicas: clase magistral, debate, trabajo y presentación en grupo. En todos los casos, los estudiantes tuvieron que tomar sus propias decisiones acerca del contenido formativo de la asignatura. Previamente, el profesor dio a conocer el propósito de la materia, los temas de la misma e investigó sobre las expectativas de los alumnos. Tras las primeras sesiones, desarrolladas por el docente, los estudiantes presentaron por equipos (de dos a tres personas) los temas elegidos. La evaluación de los alumnos se realizó considerando varios aspectos: examen de conocimientos, trabajos de exposición, participación de los alumnos y auto-evaluación realizada por los propios estudiantes. Sin embargo, en el grupo de alumnos sobre los que se aplicaron métodos no participativos, se desarrollaron técnicas tradicionales: el profesor establecía los objetivos de la asignatura, impartía el contenido y daba calificaciones individuales, sin prestar tanta atención a la relación personal con el alumno. La evaluación siguió en este caso un formato tradicional, a través de exámenes y trabajos presentados durante el curso. 


\subsection{Fuentes de información}

Para evaluar los resultados de estas prácticas docentes, se optó por suministrar a los alumnos un cuestionario, en el que se incluían los siguientes bloques de información:

Bloque I: Estilo de liderazgo del docente.

Bloque II: Clima generado en el aula.

Bloque III: Grado de asimilación de la metodología aplicada.

Bloque IV: Nivel del desempeño docente.

Bloque V: Datos identificativos y de clasificación del encuestado.

Las variables incluidas en este trabajo se recogieron en el cuestionario con las escalas y unidades de medida que se muestran en los cuadros de resultados. Para la elaboración del instrumento de obtención de información se efectuó, con carácter previo, un pretest con profesores universitarios y profesionales externos, con conocimientos y experiencia en el ámbito de la formación.

\section{CUADRO 3. Procedimiento metodológico de la investigación}

\begin{tabular}{|c|l|l|l|}
\hline FASE & \multicolumn{1}{|c|}{ ANÁLISIS } & \multicolumn{1}{c|}{ METODOLOGIA } & \multicolumn{1}{c|}{ TÉCNICA } \\
\hline Previa & $\begin{array}{l}\text { Documental y validez } \\
\text { de contenido } \\
\text { Validez de expertos }\end{array}$ & $\begin{array}{l}\text { Revisión de literatura } \\
\text { Investigación } \\
\text { cuantitativa (pretest) }\end{array}$ & $\begin{array}{l}\text { Análisis de la } \\
\text { bibliografía }\end{array}$ \\
\hline Identificación & $\begin{array}{l}\text { Definición de las } \\
\text { variables } \\
\text { Fiabilidad } \\
\text { Fiabilidad y validez de } \\
\text { constructo }\end{array}$ & $\begin{array}{l}\text { Investigación } \\
\text { cuantitativa (análisis } \\
\text { de fiabilidad global } \\
\text { inicial y validez } \\
\text { factorial) }\end{array}$ & $\begin{array}{l}\text { Alfa de Cronbach } \\
\text { Co los datos }\end{array}$ \\
\hline Evaluación & $\begin{array}{l}\text { Evaluación item-total } \\
\text { variables de las }\end{array}$ & $\begin{array}{l}\text { Investigación } \\
\text { cuantitativa }\end{array}$ & $\begin{array}{l}\text { Análisis factorial } \\
\text { exploratorio descriptivo de } \\
\text { los datos }\end{array}$ \\
\hline
\end{tabular}

Elaboración propia. 
El trabajo de campo en México se realizó en las instalaciones de la Universidad, en la última semana de febrero de 2010. En Portugal, se obtuvo la información entre los días 28 de junio y 1 de julio de 2010, y la muestra incluyó alumnos de licenciatura y postgrado. El cuadro 3 detalla la metodología de la investigación efectuada.

\section{Resultados}

\section{I. Perfil de la muestra}

El cuadro 4 recoge, en términos comparados, el perfil de los estudiantes que respondieron al cuestionario. En este análisis es de destacar, en conjunto, la mayor presencia masculina entre el alumnado (54,8 \%), así como la elevada proporción de alumnos que trabajan en empresas privadas $(83,5 \%)$.

Como se puede apreciar en el cuadro 4, los alumnos de México presentan más experiencia laboral y ocupan posiciones intermedias en su organización, cursando un postgrado con el fin de promocionarse profesionalmente. Sin embargo, en Portugal los alumnos acumulan menos experiencia, lo que parece indicar que estudian fundamentalmente con el fin de insertarse en el mercado de trabajo.

CUADRO 4. Perfil de la muestra

(datos en porcentaje y le edad, en valor medio y desviación típica)

\begin{tabular}{|c|c|c|c|c|c|c|c|}
\hline & \multicolumn{6}{|c|}{ GRUPOS DE ALUMNOS } & \multirow[b]{2}{*}{$\begin{array}{c}\text { TOTAL } \\
\text { ALUMNOS }\end{array}$} \\
\hline & $\begin{array}{c}\text { Método } \\
\text { participativo }\end{array}$ & $\begin{array}{c}\text { Método no } \\
\text { participativo }\end{array}$ & $\begin{array}{c}\text { TOTAL } \\
\text { MÉXICO }\end{array}$ & $\begin{array}{c}\text { Licenciatura } \\
\text { Portugal }\end{array}$ & $\begin{array}{c}\text { Postgrado } \\
\text { Portugal }\end{array}$ & $\begin{array}{c}\text { TOTAL } \\
\text { PORTUGAL }\end{array}$ & \\
\hline $\begin{array}{l}\text { Edad: } \\
\text { - Valor medio } \\
\text { - D. T. }\end{array}$ & $\begin{array}{c}28,4 \text { años } \\
6,35\end{array}$ & $\begin{array}{c}28,3 \text { años } \\
5,09\end{array}$ & $\begin{array}{c}28,4 \text { años } \\
5,69\end{array}$ & $\begin{array}{c}21,3 \text { años } \\
3,44\end{array}$ & $\begin{array}{c}32,3 \text { años } \\
12,84\end{array}$ & $\begin{array}{c}25,1 \text { años } \\
9,5\end{array}$ & $\begin{array}{c}30 \text { años } \\
7,27\end{array}$ \\
\hline $\begin{array}{l}\text { Género: } \\
\text { - Varón } \\
\text { - Mujer }\end{array}$ & $\begin{array}{l}56,4 \% \\
43,6 \% \\
\end{array}$ & $\begin{array}{l}65,1 \% \\
34,9 \% \\
\end{array}$ & $\begin{array}{l}61 \% \\
39 \% \\
\end{array}$ & $\begin{array}{l}50 \% \\
50 \% \\
\end{array}$ & $\begin{array}{l}17 \% \\
63 \% \\
\end{array}$ & $\begin{array}{l}46 \% \\
54 \% \\
\end{array}$ & $\begin{array}{l}54,8 \% \\
45,2 \% \\
\end{array}$ \\
\hline $\begin{array}{l}\text { ¿Trabaja } \\
\text { actualmente? } \\
\text { - Sí } \\
\text { - No }\end{array}$ & $\begin{array}{l}79,5 \% \\
20,5 \%\end{array}$ & $\begin{array}{c}90,7 \% \\
9,3 \%\end{array}$ & $\begin{array}{l}85,4 \% \\
14,6 \%\end{array}$ & $\begin{array}{l}28,2 \% \\
71,8 \%\end{array}$ & $\begin{array}{l}73,7 \% \\
26,3 \%\end{array}$ & $\begin{array}{l}45 \% \\
55 \%\end{array}$ & $\begin{array}{l}68,7 \% \\
31,3 \%\end{array}$ \\
\hline
\end{tabular}




\begin{tabular}{|c|c|c|c|c|c|c|c|}
\hline $\begin{array}{l}\text { Tipo de } \\
\text { organización: } \\
\text { - Pública } \\
\text { - Privada }\end{array}$ & $\begin{array}{l}25,6 \% \\
74,4 \%\end{array}$ & $\begin{array}{l}11,6 \% \\
88,4 \%\end{array}$ & $\begin{array}{l}18,3 \% \\
81,7 \%\end{array}$ & $\begin{array}{l}35,9 \% \\
64,1 \%\end{array}$ & $\begin{array}{l}21,1 \% \\
78,9 \%\end{array}$ & $\begin{array}{l}14 \% \\
86 \%\end{array}$ & $\begin{array}{l}16,5 \% \\
83,5 \%\end{array}$ \\
\hline $\begin{array}{l}\text { Posición en la } \\
\text { organización: } \\
\text { - Alta gerencia/ } \\
\text { dirección } \\
\text { - Mando intermedio } \\
\text { - Sin cargo directivo }\end{array}$ & $\begin{array}{l}10,3 \% \\
53,8 \% \\
35,9 \%\end{array}$ & $\begin{array}{c}2,3 \% \\
72,1 \% \\
25,6 \%\end{array}$ & $\begin{array}{c}6,1 \% \\
63,4 \% \\
30,5 \%\end{array}$ & $\begin{array}{c}2,6 \% \\
25,6 \% \\
71,8 \%\end{array}$ & $\begin{array}{l}31,6 \% \\
10,5 \% \\
57,9 \%\end{array}$ & $\begin{array}{c}27 \% \\
8 \% \\
65 \%\end{array}$ & $\begin{array}{l}14,8 \% \\
40,4 \% \\
44,8 \%\end{array}$ \\
\hline $\begin{array}{l}\text { Experiencia en el } \\
\text { puesto actual: } \\
\text { - Menos de un año } \\
\text { - De uno a tres años } \\
\text { - Más de tres años }\end{array}$ & $\begin{array}{l}7,7 \% \\
61,5 \% \\
30,8 \%\end{array}$ & $\begin{array}{c}9,3 \% \\
37,2 \% \\
53,5 \%\end{array}$ & $\begin{array}{c}8,5 \% \\
48,8 \% \\
42,7 \%\end{array}$ & $\begin{array}{c}7,7 \% \\
17,9 \% \\
74,4 \%\end{array}$ & $\begin{array}{l}21,1 \% \\
15,8 \% \\
63,1 \%\end{array}$ & $\begin{array}{l}25 \% \\
36 \% \\
39 \%\end{array}$ & $\begin{array}{l}15,3 \% \\
43,5 \% \\
41,2 \%\end{array}$ \\
\hline TOTAL ALUMNOS & 39 & 43 & 82 & 39 & 19 & 58 & 140 \\
\hline
\end{tabular}

Elaboración propia.

\subsection{Análisis realizados}

Este trabajo se inició con la selección de las variables representativas de las áreas identificadas como marco teórico de referencia, como recogen los cuadros 1 y 2. Una vez establecida la escala inicial de medición, ésta se sometió a la fase de pretest para cerrar definitivamente el cuestionario, la comprensión de las variables y la redacción de algunos ítems.

Para evitar sesgos en la estructura empírica, validar la estabilidad de las soluciones obtenidas en cada paso y generalizar los resultados más allá de la muestra obtenida, se aplicó el cuestionario en Portugal sobre el total de los alumnos entrevistados. Esta validación cruzada permite trabajar con dos muestras: una de ellas (la de Méxicol para la estimación, diagnóstico y modificación del instrumento previo de medición; y la otra (en Portugal) para la validación cruzada, como sugiere la literatura para trabajos de este tipo (Hernández et al., 2006).

El análisis realizado se inició con la identificación empírica de las variables, y consistió en apreciar la consistencia interna de la escala, a partir del Alfa de Cronbach y la correlación ítem-total del instrumento. Así, se calculó la fiabilidad global en la muestra de estimación de 82 casos, que arrojó un Alfa de 0,967, y el análisis de correlación ítem-total, cuyos valores estaban todos comprendidos entre 0,716 y 0,885 . 
Dado el carácter exploratorio de la investigación, sobre la muestra de estimación se aplicó un análisis factorial de componentes principales con rotación Varimax, que ofreció los resultados que se presentan en el cuadro 5.

\section{CUADRO 5. Análisis factorial exploratorio para el estilo de liderazgo docente}

\begin{tabular}{|c|c|c|}
\hline \multirow[b]{2}{*}{ İTEMS } & \multicolumn{2}{|c|}{$\begin{array}{l}\text { ANÁLISIS DE COMPONENTES } \\
\text { PRINCIPALES }\end{array}$} \\
\hline & COMUNALIDADES & $\begin{array}{l}\text { CARGAS } \\
\text { FACTORIALES }\end{array}$ \\
\hline $\begin{array}{l}\text { - Formas nuevas de hacer las cosas } \\
\text { - Diferentes puntos de vista } \\
\text { - Nuevas ideas } \\
\text { - Tolerar diferencias de opinión } \\
\text { - Entusiasmo del profesor } \\
\text { - El profesor ayuda a esforzarse } \\
\text { - El profesor concede elogios } \\
\text { - Confianza en alcanzar las metas } \\
\text { - Consecuencias morales y éticas de decisiones adoptadas } \\
\text { - Atender sentimientos y necesidades } \\
\text { - El profesor se relaciona personalmente } \\
\text { - El profesor trata al alumno individualmente } \\
\text { - Dedicar tiempo a enseñar y orientar }\end{array}$ & $\begin{array}{l}0.738 \\
0.792 \\
0.831 \\
0.732 \\
0.678 \\
0.807 \\
0.680 \\
0.770 \\
0.686 \\
0.724 \\
0.663 \\
0.561 \\
0.782\end{array}$ & $\begin{array}{l}0.859 \\
0.890 \\
0.911 \\
0.855 \\
0.824 \\
0.898 \\
0.825 \\
0.877 \\
0.828 \\
0.851 \\
0.814 \\
0.749 \\
0.885\end{array}$ \\
\hline $\begin{array}{l}\text { \% varianza total: } \\
\text { Medida KMO: } \\
\text { Prueba de esfericidad de Bartlett: } \\
\text { Determinante matriz de correlaciones: }\end{array}$ & \multicolumn{2}{|c|}{$\begin{array}{l}78.932 \% \\
0.942 \\
1721.480 \text { (g. I. = 105; } p<0.000) \\
1.13 E-110\end{array}$} \\
\hline
\end{tabular}

Elaboración propia.

El análisis de las cargas de las variables sobre los factores proporcionó evidencia de la interpretación conceptual subyacente en la escala de medición, con valores que superan el 0,850 en los ítems relacionados con la creatividad/experimentación (como "formas nuevas de hacer las cosas", "diferentes puntos de vista", "nuevas ideas" y "tolerar diferencias de opinión"), y fomentando la motivación de los alumnos (en "alcanzar las metas del curso", y "ayudar a esforzarse"), así como la empatía con el grupo ("atendiendo a sentimientos...", y "dedicando tiempo a enseñar y orientar a los estudiantes"). 


\section{CUADRO 6. Análisis factorial exploratorio para el desempeño docente}

\begin{tabular}{|l|c|c|}
\cline { 2 - 3 } \multicolumn{1}{c|}{} & \multicolumn{2}{c|}{$\begin{array}{c}\text { ANÁLISIS DE COMPONENTES } \\
\text { PRINCIPALES }\end{array}$} \\
\hline \multicolumn{1}{c|}{ İTEMS } & COMUNALIDADES & $\begin{array}{c}\text { CARGAS } \\
\text { FACTORIALES }\end{array}$ \\
\hline - Utilizar métodos didácticos satisfactorios & 0.756 & 0.869 \\
- Utilizar el buen humor & 0.820 & 0.905 \\
- Satisfacción con los logros del grupo & 0.804 & 0.897 \\
- Satisfacción al cumplir con lo esperado & 0.716 & 0,846 \\
- Satisfacción global & 0.826 & 0.909 \\
- Motivar para trabajar más duro & 0.738 & 0.859 \\
- Hacer más de lo que esperaba & 0.752 & 0.867 \\
- Orgullo por el trabajo bien hecho & 0.829 & 0.910 \\
- Recompensa en alcanzar las metas & 0.855 & 0.925 \\
- Motivación general por tener éxito & 0.837 & 0.915 \\
- Buscar formas de motivar al grupo & 0.832 & 0.912 \\
- Satisfacer las necesidades del grupo & 0.863 & 0.929 \\
- Asignar tareas para lograr objetivos & 0.798 & 0.893 \\
- Ayudar siempre que se necesite & 0.653 & 0.808 \\
- Satisfacción general con los objetivos del curso & 0.762 & 0.873 \\
\hline \% varianza total: & $72.636 \%$ & \\
Medida KMO: & 0.940 & \\
Prueba de esfericidad de Bartlett: & 1134.979 (g. I. = $78 ; \mathrm{p}<0.000$ ) \\
Determinante matriz de correlaciones: & $3.16 E-007$ \\
\hline
\end{tabular}

Elaboración propia.

De la misma forma, se actuó sobre la medición del desempeño docente, que arrojó un Alfa de Cronbach de 0,981. El análisis de correlación ítem-total presentó unos valores comprendidos entre 0,782 y 0,918. La aplicación del análisis factorial de componentes principales con rotación Varimax proporcionó los resultados que se aprecian en el cuadro 6, y las cargas de las variables sobre los factores ofrecieron valores superiores al 0,850 en casi todos los ítems relacionados con la satisfacción de los alumnos con el desempeño del profesor (como el "de métodos didácticos satisfactorios", "utilizar el buen humor en las clases" y "alcanzar la satisfacción con los logros del grupo"). Lo mismo cabe decir de rasgos referidos a exigir un esfuerzo extra a los alumnos (como el "motivar para trabajar más duro", "hacer más de lo que se esperaba", "tener orgullo por el trabajo bien hecho", "recompensar por las metas conseguidas" y "motivación general por tener éxito"), y alcanzar la efectividad en lograr los resultados de aprendizaje esperados (con "nuevas 
formas de motivar al grupo", "satisfacer sus necesidades", "asignar tareas entre los miembros del grupo" y "satisfacción general con los objetivos del curso").

\subsection{Interpretación de los resultados}

Puesto que una finalidad de este trabajo, de carácter esencialmente exploratorio, fue la validación cruzada del instrumento original de medición, esta parte del análisis se efectuó sobre la muestra total de estudiantes en Portugal, tanto de grado como de postgrado.

El cuadro 7 presenta las respuestas de los estudiantes a la escala del estilo de liderazgo del profesor. En este punto es interesante resaltar las elevadas valoraciones medias del estilo participativo aplicado (la media de las respuestas se situó en valores superiores a 3: ni de acuerdo, ni en desacuerdo); resultados en línea con lo observado para este tipo de metodología (De Vincenzi, 2009; Pedraja-Rejas et al., 2009; Ramírez y Sgambatti, 2008). El análisis detallado del cuadro revela unas elevadas puntuaciones en los ítems más relacionados con la creatividad/ experimentación; en especial al "tolerar las diferencias de opinión" (media total de 3,75 y desviación típica de 0,69 ), sobre todo en los alumnos de postgrado; de "distintos puntos de vista" $(3,86$ de media total y una desviación de 0,54$)$ y "generar nuevas ideas en el aula" (con valores totales por encima del 3,60 y 0,62, en una escala de importancia de 1 -mínimo-a 5 -máximo-).

Por otra parte, son los ítems de motivación los más valorados por los alumnos (el "entusiasmo del profesor en el aula" y "dar confianza en que se alcanzarán las metas", con valores medios de 4,24 y 3,72 en la columna de total). En el bloque de cuestiones sobre el estilo de liderazgo del docente, el ítem relativo a que "el profesor tiene tendencia a hablar con entusiasmo" ha obtenido una media de respuestas de 4,24; y una desviación típica de 0,57 (columna total). Los restantes indicadores, vinculados a la motivación del profesor, tienen también medias superiores a 3 en esta columna (entre 3,57 y 3,78 ), pero con desviaciones más significativas. Además, el análisis detallado para los dos grupos de estudiantes revela el hecho de que "el profesor tiene tendencia a hablar con entusiasmo sobre las metas a alcanzar": una opinión distribuida de forma prácticamente uniforme entre los alumnos. Por su parte, en cuanto a la "conciencia del profesor sobre las consecuencias morales y éticas de las decisiones tomadas", las opiniones positivas están repartidas de forma muy parecida en los dos grupos de alumnos estudiados (como puede apreciarse en el cuadro 7). Por último, la empatía es un aspecto muy apreciado por los estudiantes, porque refleja disposición del docente con el alumno "atendiendo 
a sus sentimientos y necesidades" (3,10 de valor medio, en la columna total), y "dedicando tiempo a enseñar y orientar" $(4,07)$. Algo muy valorado es también que "el profesor se relaciona de forma personal con el estudiante" $(3,14)$, y "lo trata individualmente" $(3,72)$. En definitiva, en este bloque de ítems sobre el estilo de liderazgo, se verificó que todos ellos arrojan resultados medios por encima de 3,00 . Las opiniones neutras (con valores cercanos a 3 ) tienen más representación en el grupo de licenciatura, mientras que en los cursos de postgrado están más de acuerdo con esta postura del docente.

\section{CUADRO 7. Escala de estilo de liderazgo docente (datos en valores medios y desviaciones típicas)}

\begin{tabular}{|c|c|c|c|c|c|c|}
\hline & \multicolumn{4}{|c|}{ GRUPOS DE ALUMNOS } & \multirow{2}{*}{\multicolumn{2}{|c|}{ TOTAL }} \\
\hline & \multicolumn{2}{|c|}{$\begin{array}{l}\text { Licenciatura } \\
\text { Portugal }\end{array}$} & \multicolumn{2}{|c|}{$\begin{array}{c}\text { Postgrado } \\
\text { Portugal }\end{array}$} & & \\
\hline & Media & D. T. & Media & D. T. & Media & D. T. \\
\hline $\begin{array}{l}\text { - Formas nuevas de hacer las cosas } \\
\text { - Diferentes puntos de vista } \\
\text { - Nuevas ideas } \\
\text { - Tolerar diferencias de opinión } \\
\text { - Entusiasmo del profesor } \\
\text { - El profesor ayuda a esforzarse } \\
\text { - El profesor concede elogios } \\
\text { - Confianza en alcanzar las metas } \\
\text { - Consecuencias morales y éticas de decisiones adoptadas } \\
\text { - Atender sentimientos y necesidades } \\
\text { - El profesor se relaciona personalmente } \\
\text { - El profesor trata al alumno individualmente } \\
\text { - Dedicar tiempo a enseñar y orientar }\end{array}$ & $\begin{array}{l}3,08 \\
3,79 \\
3,62 \\
3,54 \\
4,26 \\
3,51 \\
3,64 \\
3,59 \\
3,79 \\
2,97 \\
2,92 \\
3,59 \\
4,13\end{array}$ & $\begin{array}{l}0,66 \\
0,62 \\
0,67 \\
0,64 \\
0,64 \\
0,60 \\
0,84 \\
1,19 \\
1,22 \\
0,67 \\
0,96 \\
0,68 \\
0,62\end{array}$ & $\begin{array}{l}3,95 \\
4,00 \\
3,58 \\
4,22 \\
4,21 \\
3,68 \\
3,53 \\
4,00 \\
3,74 \\
3,37 \\
3,58 \\
4,00 \\
3,95\end{array}$ & $\begin{array}{l}1,39 \\
0.33 \\
0,51 \\
0,55 \\
0,42 \\
0,75 \\
0,91 \\
0,47 \\
0,73 \\
0,90 \\
0,75 \\
1,00 \\
0,71\end{array}$ & $\begin{array}{l}3,36 \\
3,86 \\
3,60 \\
3,75 \\
4,24 \\
3,57 \\
3,60 \\
3,72 \\
3,78 \\
3,10 \\
3,14 \\
3,72 \\
4,07\end{array}$ & $\begin{array}{l}1,04 \\
0,54 \\
0,62 \\
0,69 \\
0,57 \\
0,65 \\
0,86 \\
1,02 \\
1,07 \\
0,77 \\
0,95 \\
0,81 \\
0,65\end{array}$ \\
\hline
\end{tabular}

Elaboración propia.

En una interpretación conjunta de los 4 últimos ítems del estilo de liderazgo, puede concluirse que la percepción general de los alumnos, en cuanto a su relación con el profesor, revela que éste "dedica gran parte de su tiempo a orientar y enseñar" (4,07 de media total), está "atento a cada uno de los individuos", pero sin tener demasiado en cuenta sus necesidades individuales (3,72 de media), y con una "relación" muy personalizada (valor medio de 3,14 sobre la columna total). 


\section{CUADRO 8. Escala de evaluación del desempeño docente}

(datos en valores medios y desviaciones típicas)

\begin{tabular}{|l|c|c|c|c|c|c|}
\cline { 2 - 5 } \multicolumn{1}{c|}{} & \multicolumn{3}{|c|}{ GRUPOS DE ALUMNOS } & \multicolumn{2}{c|}{} \\
\cline { 2 - 6 } \multicolumn{1}{c|}{$\begin{array}{c}\text { Licenciatura } \\
\text { Portugal }\end{array}$} & \multicolumn{2}{c|}{$\begin{array}{c}\text { Postgrado } \\
\text { Portugal }\end{array}$} & \multicolumn{2}{c|}{ TOTAL } \\
\cline { 2 - 6 } & Media & D. T. & Media & D. T. & Media & D. T. \\
\cline { 2 - 6 } - Utilizar métodos didácticos satisfactorios & 3,56 & 0,68 & 3,79 & 0,71 & 3,64 & 0,69 \\
- Utilizar el buen humor & 3,69 & 0,69 & 4,42 & 0,61 & 3,93 & 0,75 \\
- Satisfacción con los logros del grupo & 3,51 & 0,82 & 3,84 & 0,90 & 3,62 & 0,86 \\
- Satisfacción al cumplir con lo esperado & 3,46 & 0,68 & 3,74 & 0,87 & 3,55 & 0,75 \\
- Satisfacción global & 3,62 & 0,82 & 4,00 & 0,82 & 3,74 & 0,83 \\
- Motivar para trabajar más duro & 3,28 & 1,26 & 3,47 & 0,70 & 3,34 & 1,10 \\
- Hacer más de lo que esperaba & 3,26 & 1,25 & 3,32 & 0,75 & 3,28 & 1,11 \\
- Orgullo por el trabajo bien hecho & 3,51 & 1,09 & 3,53 & 0,91 & 3,52 & 1,03 \\
- Recompensa en alcanzar las metas & 3,44 & 1,14 & 3,26 & 0,87 & 3,38 & 1,06 \\
- Motivación general por tener éxito & 3,28 & 1,23 & 3,63 & 0,83 & 3,40 & 1,12 \\
- Buscar formas de motivar al grupo & 3,51 & 1,12 & 3,63 & 0,68 & 3,55 & 1,01 \\
- Satisfacer las necesidades del grupo & 3,56 & 1,09 & 3,68 & 0,82 & 3,60 & 0,94 \\
- Asignar tareas para lograr objetivos & 3,85 & 1,01 & 3,58 & 0,77 & 3,76 & 0,89 \\
- Ayudar siempre que se necesite & 4,18 & 0,94 & 4,58 & 0,51 & 4,31 & 0,71 \\
- Satisfacción general con los objetivos del curso & 3,57 & 1,11 & 4,00 & 0,75 & 3,91 & 0,99 \\
\hline
\end{tabular}

Elaboración propia.

El cuadro 8 presenta los resultados sobre la evaluación del desempeño del profesor en el aula, a través de tres conjuntos de afirmaciones (relacionadas con aspectos de satisfacción, esfuerzo extra y efectividad en el proceso de aprendizaje de los alumnos). En este caso, también se observa que los resultados en ambos casos (en los que se ensayó un estilo docente participativo: licenciatura y postgrado) son elevados para casi todos los indicadores recogidos en este trabajo. Así, en el primer conjunto de ítems, las opiniones presentan medias en general por encima de 3,55. Destacan, en especial, las opiniones más concordantes con el hecho de que la actuación del docente consigue "mejorar el clima en el aula con buen humor" (3,93 de media en la columna total), y que "el profesor utiliza métodos didácticos que me resultan satisfactorios" $(3,64)$. Como evaluación general del estilo del docente en este conjunto de ítems, las respuestas tienen un valor medio de 3,69. En este análisis, se desprende una coincidencia en las preferencias de los alumnos por estilos de tipo participativo (con modalidades de enseñanza como debates, trabajos y presentaciones en grupo), frente a la alternativa tradicional de la clase magistral, con el control del profesor de todos los elementos del proceso 
de enseñanza; en línea con los resultados de estudios previos, que enfatizan la efectividad de métodos pedagógicos en los que los alumnos son coproductores de su propio aprendizaje (Caligiore y Díaz, 2006; Escribano, 1995).

En cuanto al segundo grupo de cuestiones, relacionado con aspectos de esfuerzo extra a pedir a los alumnos, los valores obtenidos han sido más modestos (medias entre 3,28 y 3,52 ) que en el primer conjunto de datos, pero asimismo todos de opiniones mayoritariamente concordantes. El ítem con mejores resultados ha sido "el profesor me hace sentir orgullo por el trabajo bien hecho", mientras que "el profesor me motiva a hacer más de lo que esperaba hacer" ha obtenido valores más bajos (medias de 3,52 y 3,28, respectivamente). En suma, en general los alumnos se manifestaron satisfechos con la forma como perciben la metodología, y su reflejo en los resultados.

En el último conjunto de variables, las medias se sitúan por encima del 3,55 en la columna total, con mayor relevancia para el indicador "el profesor está dispuesto a ayudar siempre que se le necesite" (4,31 de media y 0,71 de desviación típica) y "satisfacción general con el cumplimiento de los objetivos del curso" 3,91 de valor medio total). En resumen, a pesar de que existe un número significativo de respuestas neutras en los 4 primeros indicadores de medición, de una forma general los alumnos están de acuerdo con que el desempeño del docente permite desarrollar "formas de motivary satisfacer las necesidades del grupo", atribuyendo "tareas para lograr los objetivos didácticos", y está "dispuesto a ayudar a lograrlos siempre que se necesite". De modo que, en términos generales, los estudiantes se manifiestan satisfechos con el cumplimiento de los objetivos del curso. En síntesis, puede afirmarse que los alumnos encuestados tienen, de una forma general, una opinión positiva sobre los aspectos estudiados. Destaca especialmente la opinión de concordancia, en cuanto al estilo de liderazgo docente, en los indicadores "el profesor ayuda a analizar los problemas según diferentes puntos de vista", "tiene tendencia a hablar con entusiasmo sobre los retos a alcanzar", "trata a cada individuo de forma personal" , y no como uno más entre otros; $y$ "dedica tiempo a enseñar y orientar". Por otra parte, en este bloque de aspectos relacionados con el estilo de liderazgo, las valoraciones más bajas se manifestaron sobre "la relación personal con cada alumno", y que el docente "presta atención a los sentimientos y necesidades de los alumnos".

El análisis detallado por grupos de alumnos permite reforzar estos resultados. En particular, en los estudiantes de licenciatura son muy valorados los indicadores relativos a la efectividad (con niveles, para el indicador de "ayudar al alumno siempre que se le necesite", de 4,18 de valor medio). En este sentido, la práctica 
del debate permitió desarrollar muchas de las competencias necesarias para una gestión eficiente del conocimiento, en especial las relacionadas con la asertividad y el aprender a escuchar; elementos esenciales para estimular capacidades de liderazgo en los alumnos.

Del análisis del cuadro 8 se desprende que, para los alumnos de grado, los ítems de evaluación del desempeño, en los rasgos relativos al esfuerzo extra, son criterios menos valorados por los estudiantes. Así y en particular, los ítems de "motivar para trabajar más duro", con la ayuda del profesor y "alcanzar la motivación general para tener éxito", obtienen valores del 3,28 (en la escala de importancia de 1 -mínimo- a 5 -máximo). En la misma línea de análisis, son muy altos los valores medios de "ayudar siempre que se necesite" $(4,18$, en la escala de medición), "utilizar el buen humor" $(3,69)$, y "satisfacción general con los objetivos del curso" $(3,57)$.

En cuanto a los rasgos relativos a la efectividad (entendida como las acciones del profesor que permiten alcanzar los objetivos del curso), el análisis de los datos revela unos valores altos para los indicadores de "utilizar métodos didácticos satisfactorios" $(3,56)$, "mostrar satisfacción con los logros del grupo" $(3,51)$ y alcanzar la "satisfacción al cumplir con lo esperado" $(3,46)$. Así, la metodología aplicada por el profesor en el aula refuerza la relevancia de criterios de evaluación del desempeño, como la cantidad y la calidad de los contenidos que se evalúan, la elaboración de conclusiones, la adecuación a los objetivos planteados, la presentación, la síntesis de contenidos y la utilización de referencias bibliográficas. Con la aplicación de la técnica del debate, son competencias desarrolladas por los estudiantes: la validez del enfoque y planteamiento aportado al resto de la clase por los alumnos, la integración entre teoría y práctica, la argumentación, la elaboración de conclusiones, la adecuación a los objetivos marcados, la claridad expositiva y la aportación de datos al análisis -con el fin de reforzar las conclusiones obtenidas-. En definitiva, resultados en línea con el desarrollo de actitudes, valores y habilidades sociales, como requerimientos de un nuevo mercado de trabajo (AC Nielsen, 2000; UNESCO, 1998). Los resultados son bastante expresivos en casi todos los ítems, destacando que "el profesor mejora el clima en el aula con buen humor", "hace sentir orgullo por el trabajo bien hecho por los alumnos" y "está dispuesto a ayudar siempre que se necesite". En definitiva, opiniones relevantes en cuanto a la satisfacción con el estilo del docente, su forma de motivación y cumplimiento de los objetivos del curso.

El análisis detallado por grupos de alumnos reafirma los resultados anteriormente expuestos. Las altas puntuaciones que se dan en postgrado (en todos los ítems menos en dos) se corresponden, en gran medida, con la aplicación de esta técnica 
en un grupo más reducido y motivado de estudiantes, en clases convertidas en seminarios y con una metodología propia de aprendizaje cooperativo.

\section{CONCLUSIONES}

Tras poner en práctica experiencias didácticas basadas en metodologías participativas en el aula, este artículo aporta un método original para evaluar, de forma sencilla y sistemática, la relevancia de un liderazgo transformacional en el desempeño del profesor en la clase. En el estudio empírico se han empleado datos obtenidos en el Marco de un Convenio Internacional, entre universidades de dos países. De toda esa información se ha obtenido una amplia representación espacial, de carácter transversal, acerca del fenómeno estudiado. En los siguientes apartados se presentan las contribuciones metodológicas de este estudio, las implicaciones prácticas, las limitaciones y las futuras líneas de investigación.

\section{I. Contribuciones metodológicas}

En cuanto a sus aportaciones metodológicas, la investigación descrita en estas páginas presenta una escala exploratoria ad hoc del estilo de liderazgo docente, operativa y fácilmente aplicable, que puede adaptarse por éste en función de las técnicas didácticas implementadas en su asignatura. Su virtud radica no solo en la envergadura del trabajo, que se ha realizado en dos países y en instituciones que desarrollan prácticas docentes basadas en competencias desde hace tiempo, sino que se ha analizado en postgrado, en alumnos con circunstancias académicas y profesionales similares, y en contextos de metodologías participativas (que fomentan el desarrollo de competencias en los egresados universitarios).

En este estudio se han evaluado asimismo las características psicométricas de las escalas de medición de dos conceptos, tanto el estilo de liderazgo del profesor como su desempeño docente, en términos de su fiabilidad, objetividad y validez de contenido, de expertos y factorial de las escalas diseñadas; mostrando que aquellos constructos pueden evaluarse a través de la selección de ítems aportados en este trabajo. La aplicación del estudio en México (para la identificación del modelo) y en Portugal (para su posterior validación) confirma la utilidad de la propuesta conceptual presentada en estas páginas. Además, el carácter innovador de este instrumento reside en preguntar directamente a los estudiantes, a diferencia de otros planteamientos, centrados en las evaluaciones de los profesores. 


\subsection{Implicaciones prácticas}

Por lo que se refiere a las implicaciones prácticas de esta investigación, este método es útil para examinar el liderazgo y el desempeño docentes como resultados formativos, así como los elementos sociales y afectivos presentes en el proceso de aprendizaje de los estudiantes. Además, este procedimiento resulta directamente aplicable en el aula, y también puede ser adoptado en distintos países. En definitiva, el conocimiento sobre estas prácticas constituye una herramienta esencial de planificación del currículum académico de los estudiantes; y resulta de interés tanto para los profesores, como para los directores de las instituciones educativas, con el fin de analizar sus propios resultados docentes.

La revisión de las prácticas de evaluación a través de este instrumento, como forma de valorar el éxito del programa de la asignatura, proporciona a los profesores información sobre cómo se hace operativo el proceso de aprendizaje de los estudiantes. Para los responsables académicos de las instituciones educativas, esta herramienta permite desarrollar propuestas de intervención a partir de conductas de liderazgo docente observadas, con el fin de fortalecer el proceso de selección del profesorado y desarrollar programas de formación, teniendo en cuenta la misión y visión institucionales.

\subsection{Limitaciones y futuras líneas de investigación}

Una característica esencial del método propuesto está relacionada con la propuesta de los indicadores de desempeño, cuyos valores pueden ir modificándose de acuerdo con los niveles y preferencias de los alumnos con cada técnica pedagógica desarrollada. Aunque esta limitación es salvable, puesto que el método se ajusta flexiblemente a los indicadores particulares establecidos por el profesor en su asignatura.

Otra limitación de orden práctico es que una comprensión global del fenómeno estudiado aconseja incorporar otros enfoques de evaluación docente, como por ejemplo: los programas de las diversas agencias regionales y nacionales de acreditación del profesorado; los procedentes de instrumentos que evalúen indicadores objetivos de rendimiento académico de los alumnos y de los profesores; o bien otros también subjetivos, pero desde la visión de otros agentes (colegas, egresados, etc), en un proceso de triangulación que complemente el énfasis afectivo de este instrumento de medición. Además, la metodología propuesta puede llevar a un cuestionamiento constante en la asignatura, que es capaz de identificar tanto 
las oportunidades como los riesgos vinculados al desarrollo de un pensamiento crítico en los alumnos; una cuestión que, por otra parte, constituye una obligación ética de la Universidad.

Por último, y en cuanto a las futuras líneas de trabajo, si bien esta herramienta se ha aplicado en postgrado y en el ámbito de la Administración y Dirección de Empresas, y de las Tecnologías de la Información, no se excluye su extensión a diferentes niveles del sistema educativo, así como en diversos países y áreas distintas de conocimiento.

Otra posible vía de estudio consiste en incorporar la perspectiva longitudinal en el análisis de los conceptos investigados, como criterio para evaluar la trayectoria académica de los docentes. En futuras investigaciones también resultará de especial interés la interacción del estilo de liderazgo del profesor con aspectos como las características del grupo que este dirige.

\section{Referencias bibliográficas}

aC Nielsen Research Services (2000) Employer Satisfaction with Graduate Skills: Research Report. Canberra, Department of Employment, Education, Training and Youth Affairs. Disponible en http://www.dest.gov.au.

AhUMADA, L., MONTECINO, C. y SISTO, V. (2008) "Desarrollo y validación de una escala para evaluar el funcionamiento del equipo directivo de los centros educativos": Revista Interamericana de Psicología, Vol. 42, n², pp. 228-235.

Antonakis, J., Avolio, B., Sivasubramaniam, N. M., Hinkelman, D. (2003) "Context and leadership: an examination of the nine-factor full-range leadership theory using the Multifactor Leadership Questionnaire": The Leadership Quarterly, Vol. 14, pp. 261-295.

Atencio, L., ArRietA, B. (2005) "El liderazgo y la toma de decisiones en las organizaciones de educación superior": Omnia, Vol. 11, n 1, pp. 1-24.

BAss, B. M.; Avouo, B. J. (1997) Full range leadership development: manual for the multifactor leadership questionnaire, CA, Mind Garden. 
BASS, B. M.; Avouo, B. J. (2000) MLQ Multifactor Leadership Questionnaire. Second Edition. Sampler Set: technical report, leader form, rater form and scoring key for MLQ Form 5XShort, CA, Mind Garden.

BENNETSS, M. S. (2007) "El liderazgo transformacional y la evaluación de programas académicos universitarios en México": Educar, n 40, pp. 163-189.

Berggren, A., Burgos, D., Fontana, J. M., Hinkelman, D. y Hung, V. (2005) "Practical and pedagogical issues for teacher adoption of IMS learning design standards in Moodle LMS": Journal of Interactive Media in Education, Vol. 2, pp. 1-24.

BIGGS, J., TANG, C. ('2007) Teaching for Quality Learning at University, Berks, Srhe and Oup.

BOBBIT, L.M., INKS, S. A., KEMP, K.J. y MAYO, D.T. (2000) "Integrating marketing courses to enhance team-based experiential learning": Journal of Marketing Education, Vol. 22, n 1, pp. 15-24.

BOYATZIS, R (1999) "Building on competence: the effective use of managerial talent": Human Resource Strategies, London, Salaman, G. Sage Pub., pp. 260-272.

BuRNS, A.C. (1990) "The use of live case studies in business education: Pros, cons y guidelines": Guide to business gaining y experiential learning, edited by James Gentry, London, Nichols / GP Publishing, pp. 201-15,

CAllgiore, I., Díaz, J. A. (2006) "Clima organizacional y desempeño de los docentes en la ULA: estudio de un caso": Gerencia, Vol. 8, n² 24, pp. 1-18.

Campos, M., Lafferière, T. y Harasim, L. (2001) "The Post-Secondary Networked Classroom: Renewal of Teaching Practices and Social Interaction": Journal of Asynchronous Learning Network, Vol. 5, pp. 36-52.

Castillo, S., Cabrerizo, S. (2003) Prácticas de evaluación educativa, Madrid, Pearson Educación.

CeRDÁ, L. M., RAmírez, M. (2010) "Evaluación de estilos de liderazgo en la docencia: una aplicación en la enseñanza universitaria de postgrado": Revista lberoamericana de Sistemas, Cibernética e Informática, Volumen 7, n 1, pp. 55-62. 
Cerdá, L. M., Ramos, C. (2011) "Un procedimiento de evaluación del estilo de liderazgo en la docencia": Actas de las XXI Jornadas Hispano-Lusas de Gestión Científica, ETEA-Universidad de Córdoba, pp. 185-200.

CuAdrado, I.; Molero, F. y NaVAs, M. (2003) "El liderazgo de hombres y mujeres: diferencias en estilos de liderazgo, relaciones entre estilos y predictores de variables de resultado organizacional": Acción Psicológica, Vol. 2, n² 2, pp. 115-129.

DAVENPORT, T. H., PRUSAK, L. (1998) Working knowledge: how organizations manage what they know, Boston, Harvard Business School Press.

De VINCENZI A. (2009) "Concepciones de enseñanza y su relación con las prácticas docentes: un estudio con profesores universitarios": Educación y educadores, Vol. $12, n^{\circ} 2$, pp. 87-101.

Dochy, F., Segers, M. Y SluIJsmans, D. (1999) "The use of self-, peer and coassessment in higher education: a review", Studies in Higher Education, Vol. 24, $n^{\circ} 3$, pp. 331-349.

EltoN, L. (2001) "Criteria for Teaching Competence and Teaching Excellence in Higher Education", en AYLETT y GREGORY (eds., 1996) Evaluating Teacher Quality in Higher Education, London, Ed. Falmer Press.

EscRIBANO, A. (1995) Aprendizaje cooperativo y autónomo en la enseñanza universitaria. Enseñanza, Vol. 13, pp. 89-102.

FEIXAS, M. (2004) "La influencia de factores personales, institucionales y contextuales en la trayectoria y el desarrollo docente de los profesores universitarios": Educar, $n^{\circ} 33$, pp. 31-59.

FrancIS, S. (2006) "Hacia una caracterización del docente "universitario" excelente: una revisión a los aportes de la investigación sobre el desempeño del docente universitario", Educación, Vol. 30, n 1, pp, 31-49.

Frontczak, N. T., Kelley, C.A. (2000) "Special issue on experiential learning in marketing education", Journal of Marketing Education, Vol. 22, n 1, pp. 3-4.

FuentES, M. T. (2007) "Las competencias académicas desde la perspectiva interconductual". Acta Colombiana de Psicología, Vol. 10, n² 2, pp. 51-58. 
GENTRY, J. W. (1990) "What is experiential learning?" In Guide to business gaming y experiential learning, edited by James Gentry, London, Nichols/GP Publishing, pp. 9-20.

Gil-García A., MuÑIz, M. y Delgado, A. (2008) "El liderazgo transformativo en el ámbito escolar: un esfuerzo de investigación en acción y cooperación entre instituciones de Educación Superior": Sapiens, Revista Universitaria de Investigación, Año 9, n 1, junio, pp. 13-33.

GonzÁlez, O., González, O. (2008) "Percepciones de los estudiantes universitarios frente al liderazgo del docente", Revista Mexicana de Orientación Educativa, Vol. V, $n^{\circ} 13$, noviembre de 2007-febrero de 2008, pp. 37-43.

GonzÁlez, O. (2008) "Liderazgo transformacional en el docente universitario: Maracaibo, Venezuela. Universidad del Zulia": Multiciencias, Vol. 8, $\mathrm{n}^{\circ} 1$, pp. $38-47$.

HAMER, L. O. (2000) "The additive effects of semistructured classroom activities on student learning: An application of classroom-based experiential learning techniques": Journal of Marketing Education, Vol. 22, n 1, pp. 25-34.

HaVnes, A., MCDOWelL, L. (Eds., 2007) Balancing dilemmas in assessment and learning in contemporary education, USA, Ed. Routledge.

Hernández, R.; Fernández-Collado, C. y Baptista, P. (2006) Metodología de la investigación, $4^{a}$ ed., México D.F., Ed. McGraw-Hill.

Hounsell, D., Falchikov, N., Hounsell, J., Klampfleitner, M., Huxham, M., Thomson, K. y BLAIR, S. (2007) Innovative assessment across the disciplines: an analytical review of the literature, Higher Education Academy. Disponible en http://www. heacademy.ac.uk.

JOSEPH, M., JOSEPH, B. (1997) "Service quality in education: a student perspective": Quality Assurance in Education, Vol. 5, n 1, pp. 15-21.

KeNNEDY, E.J., LAWTON, L. Y WALKER, E. (2001) "The case for using live cases: Shiffing the paradigm in marketing education": Journal of Marketing Education, Vol. 23, $\mathrm{n}^{\circ} 2$, pp. 145-151.

KolB, D. (1984) Experiential learning, New York, Englewood Cliffs, Prentice Hall. 
Levim, B., MarCH, J. G. (1998) "Organizational Learning": Annual Review of Sociology, n' 14 , pp. 319-340.

LI, R. y KAYE, M. (1998) "A case study for comparing two service quality measurement approaches in the context of teaching in higher education": Quality in Higher Education, Vol. 4, n², pp. 103-113.

Lowe, K., Kroeck, K. y Sivasubramaniam, N. (1996) "Effectiveness correlates of transformational and transactional leadership: a meta-analytic review of the MLQ literature": Leadership Quarterly, Vol. 7, n 3, pp. 385-425.

LUPANO, M. L., CASTRO, A. (2008) "Estudios sobre el liderazgo. Teorías y evaluación": Psicodebate 6. Psicología, Cultura y Sociedad, Vol. 17, n 3, pp. 107-122.

MANDell, B., PheRWANI, S. (2003) "Relationship between emotional intelligence and transformational leadership style: a gender comparison": Journal of Business and Psychology, Vol. 17, n 3, Spring, pp. 387-404.

Mbawmbaw, J., Rivera, M. E., Valentín, N., Téllez, R. y Nieto, R. (2006) “Dimensiones del liderazgo docente": Investigación universitaria multidisciplinaria, Año 5, n' 5, Dic, pp.70-79.

Muenjohn, N.; Armstrong, A. (2008) "Evaluating the Structural Validity of the Multifactor Leadership Questionnaire (MLQ): Capturing the Leadership Factors of Transformational-transactional Leadership": ContemporaryManagementResearch. Vol. 4, n 1, March, pp.3-14.

MURILLO, F. J. (2006) "Una dirección escolar para el cambio: del liderazgo transformacional al liderazgo distribuido": Revista Electrónica Iberoamericana sobre Calidad, Eficacia y Cambio en Educación, Vol. 4, n monográfico, pp. 11-24.

Moss, S., RitossA, D. (2007) "The Impact of Goal Orientation on the Association between Leadership Style and Follower Performance, Creativity and Work Attitudes": Leadership, Vol. 3, n 4, pp. 433-456.

NAVARIDAS, F. (2004) Estrategias didácticas en el aula universitaria, Logroño, Ed. Publicaciones de la Universidad de La Rioja.

NonAKA, I. (1995) "The Knowledge-Creating Company", Harvard Business Review, Vol. 69, pp. 96-104.

Nunnaluy, J. C. (1987) Teoría psicométrica, México D. F., Ed. Trillas. 
PADILA, Ma T. (2002) Técnicas e instrumentos para el diagnóstico y la evaluación educativa, Madrid, Ed. CCS.

Parker, J. D., Summerfeldt, L. J., Hogan, M. J., y Majeski, S. A. (2004) "Emotional Intelligence and Academic Success: Examining the transition from high school to university": Personality and Individual Differences, Vol. 36, n 1, pp. 163-172.

Pascual, R., Villa, A., Auzmendi, E. (2006) El liderazgo transformacional en los centros docentes, Bilbao, Mensajero.

Pedraja-Rejas, L., Rodriguez-Ponce, E., Barreda, M., Sagredo, O., Y Segovia, C. (2009) "Estilos de liderazgo y resultados del sistema de medición de la calidad de la educación: un estudio empírico en los colegios básicos de la ciudad de AricaChile": Ingeniare, Revista Chilena de Ingeniería, Vol. 17, n 1, pp. 21-26.

Pérez, A., Ramos, G. y López, E. (2009) "Diseño y análisis de una escala para la valoración de la variable clima social aula en alumnos de Educación Primaria y Secundaria": Revista de Educación, n 350, septiembre-diciembre, pp. 221-252.

Polanyl, M. (1996) The Tacit Dimension, London, Ed. Routledge and Kegan.

Prahalad, C. K., Hamel, G. (1994) "Strategy as a Field of Study: Why Search for a New Paradigm?": Strategic Management Journal, Vol. 15, summer, pp. 5-16.

PRIETO, P. (2006) "El compromiso de la alta dirección en el desarrollo del liderazgo en la empresa": Economistas, n 106, noviembre, pp. 6-11.

RAmirez, J., SgAmbati, V. (2008) "Liderazgo transformacional y transaccional en estudiantes de postgrados en gerencia del Área Metropolitana de Caracas": Visión Gerencial, año 7, n² 2, jul-dic, pp. 403-416.

RAMSDEM, P. (1991) "A performance indicator of teaching quality in Higher Education: the course experience questionnaire $(C E Q)^{\prime \prime}$ : Studies in Higher Education, Vol. 16, pp. 129-150.

UNESCO (1998). La educación superior en el siglo XXI: visión y acció, Documento de trabajo, París, Conferencia Mundial sobre la Educación Superior.

ZabalZA, M. (2000) "El papel de los departamentos universitarios en la mejora de la calidad de la docencia": Revista interuniversitaria de Formación del Profesorado, Vol. 52, n⿳3 38, agosto, pp. 47-66. 